\title{
OPIOID USE DISORDER
}

nature reviews disease primers
Opioid use disorder (OUD) is a chronic relapsing disorder that is associated with a substantial global health burden.

\section{MECHANISMS}

Most data on the mechanisms of OUD are from studies using animal models of addiction. Opioid addiction is a compulsion to use an opioid, loss of control in limiting drug use and the development of a negative emotional state when drug is unavailable. A three-stage cycle of opioid addiction has been proposed: binge/intoxication, withdrawal/ negative affect and preoccupation/anticipation. These stages involve alterations in several processes and are mediated by several different regions of the brain, including the basal ganglia, amygdala and the prefrontal cortex.

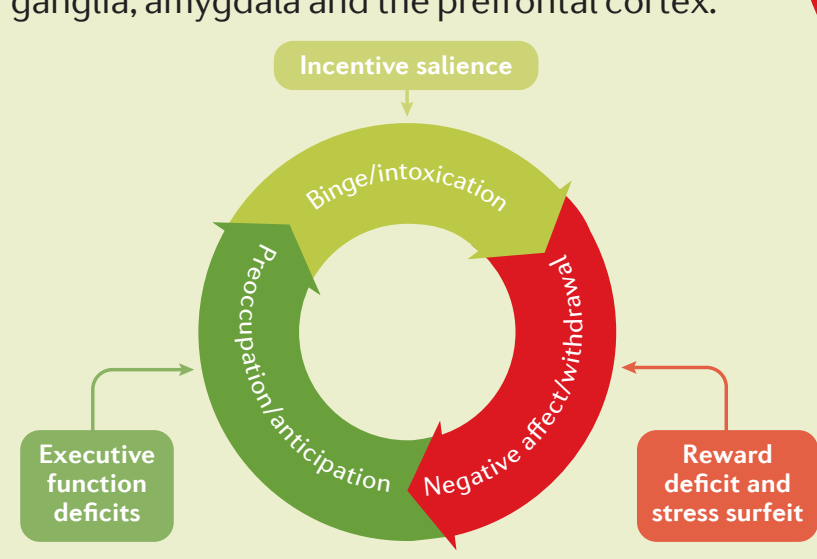

\section{DIAGNOSIS}

Formal diagnostic criteria for OUD can be found in the Diagnostic and Statistical Manual of Mental Disorders and the International Classification of Diseases. Individuals with OUD can become known to clinical services through self-referral, or after a situation whereby their drug use becomes evident, such as following overdose, interactions with the criminal justice system or diagnosis of HIV infection or hepatitis virus infection.

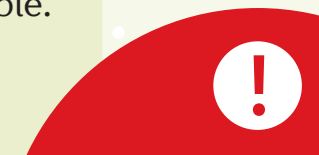

In 2016, 26.8 million individuals were estimated to have OUD worldwide, of which, the highest prevalence
horldwide, of which, the was in the USA

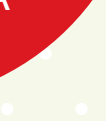

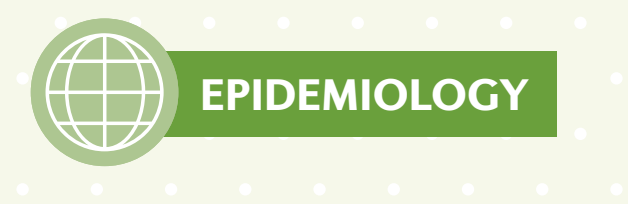
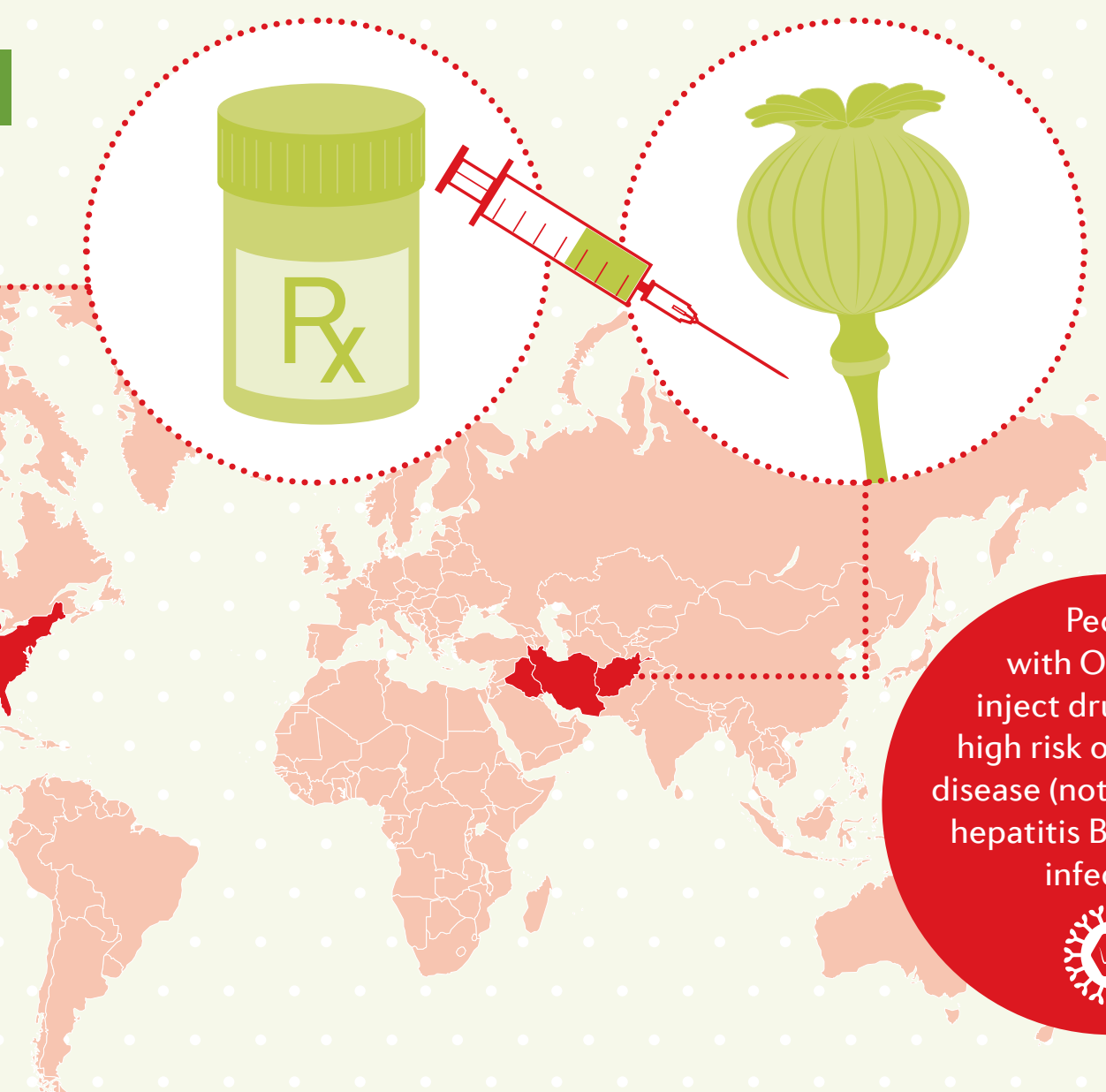

The types of opioids used in globally. For example, opium smoking is common in some Middle Eastern countries whereas misuse of prescription opioids is common in North America. Heroin is the main cause of overdose mortality in many parts of
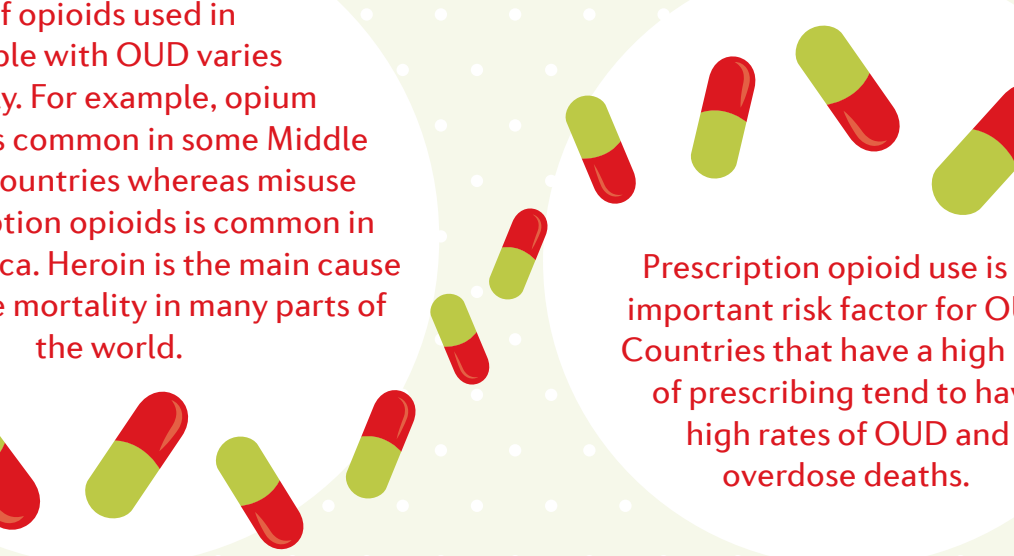

Prescription opioid use is an important risk factor for OUD. Countries that have a high rate of prescribing tend to have high rates of OUD and overdose deaths.

\section{(1) OUTLOOK}

Use of heroin and synthetic opioids — such as fentanyl — is increasing in the USA

Although effective treatments for OUD are available, access to these treatments is severely limite worldwide. This restriction is, in part, caused by under-funding and discuss the benefits of treatment strong political and public opinions with the general public and policy regarding drug use. It is imperative makers, to improve understanding that scientists and clinicians

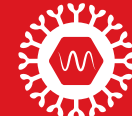

Other risk factors for OUD include peers who use drugs, mood or other genetics, male sex, negative family factors (such as childhood maltreatment and parental conflict) and low educational attainment. psychiatric disorders,

For Primervist do: 101038/511572-019-0137-5

\section{MANAGEMENT}

Opioid agonist treatments (methadone and buprenorphine) can suppress symptoms of withdrawal, reduce drug craving and block the effect of non-prescribed opioids. Antagonist treatment (naltrexone) can also be used to treat OUD but is rarely prescribed owing to difficultie with initiation of therapy and low compliance. Some individuals with OUD may benefit from attendance of mutual-aid groups, such as Narcotics Anonymous, although few studies have assessed the benefits of these groups. Behavioural therapies, such as cognitive behavioural therapy, can also be beneficial in some individuals. Education about the risk of overdose death and providing training for overdose management is vital for those with OUD.

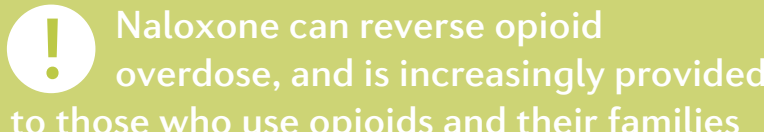
to those who use opioids and their families for interim emergency care

PREVENTION

Strategies to prevent opioid use, such as

universally restricting drug access or increasing drug cost, are difficult and costly to implement. Owing to the strong association between use of prescription opioids and OUD, several associations have reformulated guidelines for the use of opioid analgesics for chronic noncancer pain, and suggest the use of non-opioid therapies when possible.

Public health approaches to reduce distribution of sterile injecting equipment, the detection and treatment of infectious diseases, and the provision of naloxone 